\title{
Blockade of Endogenous Opioid Neurotransmission Enhances Acquisition of Conditioned Fear in Humans
}

\author{
Falk Eippert, ${ }^{1}$ Ulrike Bingel, ${ }^{2}$ Eszter Schoell, ${ }^{1}$ Juliana Yacubian, ${ }^{1}$ and Christian Büchel ${ }^{1}$ \\ Departments of ${ }^{1}$ Systems Neuroscience and ${ }^{2}$ Neurology, University Medical Center Hamburg-Eppendorf, 20246 Hamburg, Germany
}

\begin{abstract}
The endogenous opioid system is involved in fear learning in rodents, as opioid agonists attenuate and opioid antagonists facilitate the acquisition of conditioned fear. It has been suggested that an opioidergic signal, which is engaged through conditioning and acts inhibitory on unconditioned stimulus input, is the source of these effects. To clarify whether blockade of endogenous opioid neurotransmission enhances acquisition of conditioned fear in humans, and to elucidate the neural underpinnings of such an effect, we used functional magnetic resonance imaging in combination with behavioral recordings and a double-blind pharmacological intervention. All subjects underwent the same classical fear-conditioning paradigm, but subjects in the experimental group received the opioid antagonist naloxone before and during the experiment, in contrast to subjects in the control group, who received saline. Blocking endogenous opioid neurotransmission with naloxone led to more sustained responses to the unconditioned stimulus across trials, evident in both behavioral and blood oxygen level-dependent responses in pain responsive cortical regions. This effect was likely caused by naloxone blocking conditioned responses in a pain-inhibitory circuit involving opioid-rich areas such as the rostral anterior cingulate cortex, amygdala, and periaqueductal gray. Most importantly, naloxone enhanced the acquisition of fear on the behavioral level and changed the activation profile of the amygdala: whereas the control group showed rapidly decaying conditioned responses across trials, the naloxone group showed sustained conditioned responses in the amygdala. Together, these results demonstrate that in humans the endogenous opioid system has an inhibitory role in the acquisition of fear.
\end{abstract}

Key words: fear conditioning; opioids; amygdala; human; functional magnetic resonance imaging; naloxone

\section{Introduction}

One way through which animals learn about stimuli that predict danger is fear conditioning. In fear conditioning, the animal is exposed to an initially neutral stimulus [the conditioned stimulus (CS)], which is paired with an aversive stimulus [the unconditioned stimulus (US)]. As the animal learns that the CS predicts the US, the CS acquires aversive properties and is able to elicit conditioned fear responses. An important brain structure in fear conditioning is the amygdala, where convergence of CS and US information leads to synaptic plasticity and initiates conditioned responses such as freezing (LeDoux, 2000; Maren and Quirk, 2004).

Part of the repertoire of conditioned responses is the production of conditioned hypoalgesia. As the animal learns that the CS predicts the US, it becomes less pain sensitive when being exposed to the CS. This conditioned hypoalgesia is to a great part mediated by endogenous opioid neurotransmission as it can be blocked by administration of opioid antagonists (Fanselow and Baackes, 1982; Helmstetter and Fanselow, 1987) (but see Foo and

Received Dec. 2, 2007; revised March 2, 2008; accepted April 4, 2008.

This work was supported by Deutsche Forschungsgemeinschaft Grant BU 1323. We thank Jan Gläscher, Fred J. Helmstetter, Raffael Kalisch, and Arne May for helpful discussions, Thomas Rau for help with the naloxone protocol, and Hilke Andresen for analyzing the plasma concentration of naloxone.

Correspondence should be addressed to Falk Eippert, Department of Systems Neuroscience, University Medical Center Hamburg-Eppendorf, Martinistrasse 52, 20246 Hamburg, Germany. E-mail: f.eippert@uke.uni-hamburg.de. DOI:10.1523/JNEUROSCI.5336-07.2008

Copyright $\odot 2008$ Society for Neuroscience $\quad$ 0270-6474/08/285465-08\$15.00/0
Westbrook, 1994; Good and Westbrook, 1995). Opioidergic neurotransmission not only impacts on US processing, but also on CS-US association formation, as previous studies in rodents have shown that opioid agonists attenuate (Davis, 1979; Westbrook et al., 1991) and opioid antagonists facilitate (Westbrook et al., 1991; Young and Fanselow, 1992; McNally et al., 2004a) acquisition of conditioned fear. Critically, these effects are not mediated by opioid receptors in the periphery, but by opioid receptors in the CNS (Calcagnetti et al., 1987; Fanselow et al., 1988). One model assumes that endogenous opioid neurotransmission influences fear conditioning by instantiating a conditioninginduced amygdala/brainstem-dependent inhibitory signal that acts on ascending US processing (Fanselow, 1998). By attenuating the impact of the US, which is the signal that supports conditioning, this opioidergic signal regulates the acquisition of conditioned fear in a negative-feedback manner. Evidence for this conditioned opioidergic inhibitory signal is provided by studies in rodents which show that blocking opioidergic neurotransmission in structures of the aforementioned circuit (amygdala, periaqueductal gray, rostral ventromedial medulla) inhibits the development of conditioned hypoalgesia (Bellgowan and Helmstetter, 1998; Foo and Helmstetter, 1999).

In humans, the role of endogenous opioids in conditioned fear and defensive responses is mostly unknown. Although there is evidence for stress-induced hypoalgesia that can be blocked by the opioid antagonist naloxone (Willer et al., 1981; Pitman et al., 1990), a naloxone-dependent enhancement of conditioned fear 
has not been demonstrated. To elucidate the role of endogenous opioid neurotransmission in human fear conditioning, we used functional magnetic resonance imaging (fMRI) in combination with behavioral recordings and administration of the opioid antagonist naloxone. We expected that naloxone would maintain unconditioned responses to the US by blocking processing in the circuit responsible for generating the inhibitory signal that leads to the development of conditioned hypoalgesia. Furthermore, we expected that this would enhance the acquisition of conditioned fear and would influence processing in the amygdala, in that naloxone would specifically inhibit the decay of conditioned responses in the amygdala over time (Quirk et al., 1997; Büchel et al., 1998; LaBar et al., 1998) and would lead to sustained conditioned responses.

\section{Materials and Methods}

Subjects. Thirty-two male volunteers (mean age, 25.43 years; range, $18-39$ years) were assigned to two groups on a randomized double-blind basis. The experimental group received the opioid antagonist naloxone, whereas the control group received saline. We studied only male subjects to exclude possible gender effects, which have been observed in both fear conditioning and endogenous opioid function (Zubieta et al., 2002; Milad et al., 2006). The two groups did not show significant differences in age, weight, or trait anxiety (as measured with the State Trait Anxiety Inventory). Two subjects were excluded because of movements of $>2$ $\mathrm{mm}$, which left 15 subjects in the experimental group and 15 subjects in the control group. The study was approved by the Ethics Committee of the Medical Board in Hamburg, Germany, and all subjects gave written informed consent.

Drug administration. At $\sim 15$ min before the experiment, we administered a bolus dose of $0.15 \mathrm{mg} / \mathrm{kg}$ naloxone (Naloxon-ratiopharm; Ratiopharm) or saline intravenously. This dose has shown reliable naloxone effects in previous studies (Amanzio and Benedetti, 1999). However, because naloxone has a relatively short half-life $(\sim 70 \mathrm{~min}$ in blood plasma, according to the Summary of Product Characteristics, Ratiopharm) and its clinically effective duration of action can be even shorter (Gutstein and Akil, 2006), we also administered an intravenous infusion dose of $0.075 \mathrm{mg} / \mathrm{kg}$ naloxone or saline for $1 \mathrm{~h}$, starting $\sim 4 \mathrm{~min}$ after bolus administration. Supplemental Figure 2 (available at www.jneurosci.org as supplemental material) shows the plasma concentration of naloxone obtained with this protocol in five additional volunteers. Naloxone plasma concentration [which correlates strongly with concentration in brain tissue (Tepperman et al., 1983)] only decayed slightly over the course of the experiment and was still well above zero at the end of the experiment.

Study design. First, subjects underwent a urine drug screening to exclude opiate use. All subjects showed negative test results. Subjects were then given the instructions (subjects were not informed about the contingency between the CS and US and were specifically told that their response speed in the reaction time task would not influence delivery of the painful stimulus) and completed some practice trials in front of a computer in which no painful stimulus was administered and different geometric forms were used. A medical doctor (who was also blind as to whether naloxone or saline was given) then administered a bolus dose of naloxone or saline. Subjects were placed in the MR scanner and the infusion of naloxone or saline was started. Afterward, the thermal stimulator (a $30 \times 30 \mathrm{~mm}$ Peltier device, TSAII; Medoc) was placed on each subject's left volar forearm and their pain threshold was assessed. Pain threshold estimation was based on five thermal stimuli that slowly increased in temperature $\left(1^{\circ} \mathrm{C}\right.$ per second) until stopped via button press or the maximum temperature of $52^{\circ} \mathrm{C}$ was reached. To familiarize subjects with the painful heat stimulation and with the pain-rating procedure, we then applied two stimuli of 46,48 , and $50^{\circ} \mathrm{C}$ each (with a plateau duration of $1 \mathrm{~s}$ ) in a randomized pattern and asked subjects to rate the intensity of each stimulus on a visual analog scale (VAS), which ranged from 0 (no pain) to 100 (unbearable pain). The VAS consisted of two white vertical lines representing the two endpoints of the scale, and a red horizontal bar that subjects could expand to the right or compress to the left via button presses on a computer mouse to indicate the experienced pain intensity.

Afterward, the experimental paradigm started (using Presentation software; Neurobehavioral Systems), which consisted of 96 trials (48 CS +, 48 CS - ) and lasted $\sim 35 \mathrm{~min}$. We used a 50\% partial reinforcement scheme (i.e., only $50 \%$ of CS + presentations were paired with the US) Simple colored geometric forms (blue or green triangle and pentagon) on a gray background were used as the CS. The assignment of color and form to CS + and CS - (i.e., green triangle and blue pentagon) was counterbalanced across subjects. Thermal stimulation at $50^{\circ} \mathrm{C}$ to the left volar forearm was used as the US. Trial order was randomized for each subject with the limitations that (1) similar trial types were not allowed to occur more than two times consecutively and that (2) in each quarter of the experiment $12 \mathrm{CS}-, 6 \mathrm{CS}+_{\text {paired }}$, and $6 \mathrm{CS}+{ }_{\text {unpaired }}$ were presented. All visual stimuli were presented on a screen at the end of the scanner bore, which subjects could see via a tilted mirror mounted on the head coil.

Each trial began with the presentation of a CS, which remained on the screen for $10 \mathrm{~s}$. Subjects performed a reaction time (RT) task at the beginning of each trial, by reporting via button presses on a computer mouse in which half of the screen the CS was presented. In CS + paired trials, the US was administered at $8.5 \mathrm{~s}$ and coterminated with the CS (supplemental Methods and Results, available at www.jneurosci.org as supplemental material). In CS $+_{\text {unpaired }}$ trials and CS - trials, no US was administered. After CS presentation, a VAS appeared, similar to the one in the pre-experimental pain challenge, on which subjects had to rate the level of pain experienced on that trial; the rating was self-paced and only ratings given on $\mathrm{CS}+{ }_{\text {paired }}$ trials were included in the analysis. Importantly, the starting point of the red bar was completely randomized to control for motor confounds on different trial types. A variable intertrial interval (4-6 s) followed the pain rating and 16 null events of $18 \mathrm{~s}$ duration were randomly inserted into the paradigm.

After the experiment, subjects' awareness of the contingency was assessed (supplemental Methods and Results, available at www. jneurosci.org as supplemental material). Only eight subjects were aware of the contingency and there were no significant group differences. Finally, subjects were debriefed and paid for their participation.

Data acquisition. Skin conductance responses (SCRs) were acquired using $\mathrm{Ag} / \mathrm{AgCl}$ electrodes (Red Dot monitoring electrode; $3 \mathrm{M}$ Health Care) attached to the hypothenar of the subjects' left hands. The same dermatome (C8) was chosen for both electrodes to control for possible recording differences between dermatomes. We used a CED 2502 to amplify the skin conductance signal, a CED micro1401 mkII to digitize the signal at $100 \mathrm{~Hz}$, and Spike2 software to record and store the data (all equipment by Cambridge Electronic Design).

fMRI data were acquired on a 3 Tesla system (Trio; Siemens) equipped with an eight-channel head coil. Thirty-eight transversal slices (slice thickness, $2 \mathrm{~mm}$; $1 \mathrm{~mm}$ gap) were acquired in each volume (repetition time, $2230 \mathrm{~ms}$; echo time, $25 \mathrm{~ms}$; flip angle, $80^{\circ}$; field of view, $192 \times 192$ $\mathrm{mm}$; matrix, $64 \times 64)$ using $\mathrm{T}^{*}$-weighted echo-planar imaging $(\mathrm{EPI})$. Slice orientation was tilted by $-30^{\circ}$ which allowed us to measure blood oxygen level-dependent (BOLD) responses in areas as ventral as the medulla oblongata, but did not allow recording of BOLD responses in very dorsal cortical areas. The first five volumes of each subject were discarded before image analysis to exclude T1 saturation effects. We also acquired high-resolution $(1 \times 1 \times 1 \mathrm{~mm}$ voxel size $)$ T1-weighted anatomical images for each subject using a three-dimensional FLASH sequence.

Behavioral data analysis. All behavioral data were analyzed in Matlab 7.3 (MathWorks), using a threshold of $p<0.05$ one-tailed. Group differences in mean pain ratings were tested using a two-sample $t$ test. To test for habituation in the pain ratings over time, we first calculated a regression for each subject with time as predictor. We then Fisher's $z$ transformed the ensuing standardized regression coefficients ( $\beta$ weights) of each subject and used a one-sample $t$ test to test for a significant habituation effect (i.e., negative regression coefficients). To test for group differences in habituation in the pain ratings over time, we used a twosample $t$ test on the Fisher's $z$-transformed standardized regression coefficients.

Before statistical analysis of RT data, trials in which subjects missed to 
respond or responded incorrectly were discarded and the remaining data were $\mathrm{z}$ transformed. We then calculated a two-way ANOVA with the within-subject factor trial type (CS+ vs CS - ) and the between-subject factor group (naloxone vs control) and tested for a main effect of trial type and a group by trial type interaction.

SCR could not be acquired from one subject because of technical problems. Data from the remaining subjects were resampled to $10 \mathrm{~Hz}$, smoothed using a $1 \mathrm{~s}$ [full width at half maximum, (FWHM)] Gaussian kernel and $\mathrm{z}$ transformed. With regard to CS processing, we analyzed second interval responses, i.e., responses in a time window of 5-10 s after CS onset. Whereas first interval responses are generally considered CSorienting responses, second interval responses are related to US anticipation and reflect learning of the contingency (Wolter and Lachnit, 1993; Knight et al., 2003). We only analyzed responses on CS $+_{\text {unpaired }}$ trials, to exclude possible influences of the US on CS $+_{\text {paired }}$ trials. It should be noted that this in contrast to the analysis of reaction time data, in which we used all CS + trials, as reaction times occurred at CS onset and could thus not be confounded by US influences. With regard to US processing, we analyzed responses on CS ${ }_{\text {paired }}$ trials in a time window of 8.5-15 s after CS onset. Amplitudes were determined as the maximum in the analysis interval in relation to a preceding minimum in the analysis interval. Similar to the analysis of reaction time data we calculated a twoway ANOVA with the within-subject factor trial type $\left(\mathrm{CS}+{ }_{\text {unpaired }}\right.$ vs CS -) and the between-subject factor group (naloxone vs control) and tested for a main effect of trial type and a group by trial type interaction. The analysis for habituation of SCR to the US was conducted in the same way as for the pain ratings.

fMRI data analysis. fMRI data processing and statistical analyses were performed using statistical parametric mapping (SPM5, Wellcome Department of Imaging Neuroscience). Data processing consisted of slice timing (correction for differences in slice acquisition time), realignment (motion correction), spatial normalization to a standard EPI template (including resampling at a resolution of $2 \times 2 \times 2 \mathrm{~mm}$ ) and smoothing using an $8 \mathrm{~mm}$ (FWHM) isotropic Gaussian kernel. Data were also subjected to high-pass filtering (cutoff period, $128 \mathrm{~s}$ ) and correction for temporal autocorrelations (based on a first order autoregressive model).

Data analysis was performed using a general linear model approach. We modeled two types of CS responses, an early (CS onset) response directly at CS onset and a late (US anticipatory) response at $5 \mathrm{~s}$ after CS onset. On the first level, separate regressors were defined for each of the following events: CS + early, CS - early, CS + late, CS - late, US, and button presses (reaction time as well as rating button presses). In addition, we defined three regressors that represented time by event interactions. These were created (1) by multiplying the CS + early and CS - early regressor with a mean corrected exponentially decaying function with a time constant of one-fourth of the experiment length and (2) by multiplying the US regressor with a mean corrected linearly increasing function. The first-level design matrix of each subject thus consisted of nine regressors. All events were modeled as $\delta$ functions at event onset and were convolved with a canonical hemodynamic response function. After model estimation, we defined linear contrasts testing for (1) early conditioning effects (CS+ early vs CS - early), (2) late conditioning effects (CS + late vs CS - late), and (3) effects of the US. In addition, we defined contrasts testing for (1) time by condition interaction effects (CS+ early decaying vs CS - early decaying) and (2) time-dependent effects of the US. After model estimation, the ensuing first level contrast images from each subject were raised to the second level. Two-sample $t$ tests (including nonsphericity correction for possible unequal variances of the error term in the two groups) were used to test for differential group effects as well as for main effects.

To characterize the poststimulus time course of the BOLD response, we created a second model using finite impulse response (FIR) basis functions with a bin width of $2 \mathrm{~s}$ and 12 bins per trial, modeling CS + and CS - conditions separately. This basis set considers each time bin after stimulus onset individually to model the BOLD response and can capture any possible shape of response function up to a given frequency limit. In this model, the parameter estimate for each time bin represents the average BOLD response at that time. Furthermore, to investigate possible coupling between the periaqueductal gray and rostral ventromedial me-

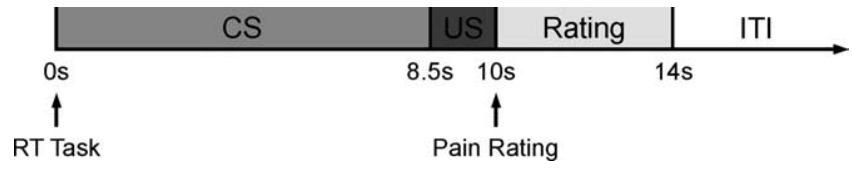

Figure 1. Experimental design. Subjects were assigned in a double-blind manner to two groups: the experimental group received the opioid antagonist naloxone before and during the experiment, whereas the control group received saline. Subjects underwent a classical fearconditioning paradigm in which two simple geometric stimuli (blue triangle and green pentagon) served as the CS. Each CS was presented for $10 \mathrm{~s}$. One stimulus (CS+) was paired with the US (heat pain delivered to left forearm at $8.5 \mathrm{~s}$ after $\mathrm{CS}$ onset) in $50 \%$ of its presentations. After each CS presentation, subjects rated the intensity of pain present on that trial on a VAS and a variable intertrial interval (ITI) followed. Subjects additionally performed an RT task at the onset of CS presentation in that they indicated whether the $C S$ was presented in the left or right half of the screen. SCRs CRs to CS and US were recorded and BOLD responses were investigated with $\mathrm{fMRI}$ at three time points: early conditioned responses at CS onset, late conditioned responses $5 \mathrm{~s}$ after $C S$ onset, and unconditioned responses at $8.5 \mathrm{~s}$ after $C S$ onset on CS + trials.

dulla, we performed a group by coupling analysis (Heinz et al., 2005) using the periaqueductal gray peak voxel as the seed region. This analysis is similar to a psycho-physiological interaction analysis (Friston et al., 1997), with the difference that it considers the whole time series of each subject and does not incorporate a psychological variable at the first level. Instead, the first-level design matrix of each subject only consists of the time course of the seed region. The resulting contrast images were then raised to the second level, where group entered as modulatory variable (two-sample $t$ test with appropriate nonsphericity correction).

In all conducted analyses, we used an initial height threshold of $p<$ 0.005 (uncorrected) and subsequent small volume correction in a priori regions of interest at a level of $p<0.05$. With regard to CS processing, these regions comprise the amygdala, the orbitofrontal cortex (OFC), the rostral anterior cingulate cortex (rACC), the periaqueductal gray matter (PAG), and the rostral ventromedial medulla (RVM), which have been implicated in conditioned fear and ensuing endogenous antinociception. With regard to US processing, these regions additionally comprise the thalamus, basal ganglia, insula, dorsal anterior cingulate cortex (dACC), dorsolateral prefrontal cortex (DLPFC), and primary and secondary somatosensory cortex, all of which have been extensively implicated in pain processing. Correction was based on peak coordinates (ignoring laterality) obtained from previous studies on fear conditioning and pain processing: the amygdala (LaBar et al., 1998), the PAG (Bingel et al., 2006), and the RVM (Fairhurst et al., 2007) were corrected using spheres of 6 $\mathrm{mm}$ radius, whereas the rACC (Wager et al., 2007), dACC (Büchel et al., 2002), OFC (Gottfried and Dolan, 2004), basal ganglia (Sprenger et al., 2006), and thalamus (Bingel et al., 2007) were corrected using spheres of $8 \mathrm{~mm}$ radius and the primary somatosensory cortex (Bingel et al., 2006), secondary somatosensory cortex (Bingel et al., 2007), insula (Bingel et al., 2007), and DLPFC (Wager et al., 2007) were corrected using spheres of 12 $\mathrm{mm}$ radius.

\section{Results}

We first investigated on the behavioral level whether our paradigm (Fig. 1) resulted in successful conditioning and development of conditioned hypoalgesia across all subjects. Significant conditioning effects were evident both in RT (faster responses to $\mathrm{CS}+$ than to CS $-; F_{(1,28)}=2.93 ; p=0.05$ ) and SCR (stronger responses to $\mathrm{CS}+$ than to $\mathrm{CS}-; F_{(1,27)}=3.57 ; p=0.03$ ). Conditioned hypoalgesia also developed, as evidenced by significant habituation of pain ratings $\left(t_{(29)}=2.76 ; p=0.01\right)$ and SCR to the US $\left(t_{(28)}=3.96 ; p=0.0005\right)$ over the course of the experiment.

\section{US processing}

Across all subjects, the US was perceived as moderately painful $[65.50 \pm 3.44$ (mean $\pm \mathrm{SE}$ ) on a scale from 0 (no pain) to 100 (unbearable pain)] and elicited responses in several pain sensitive 

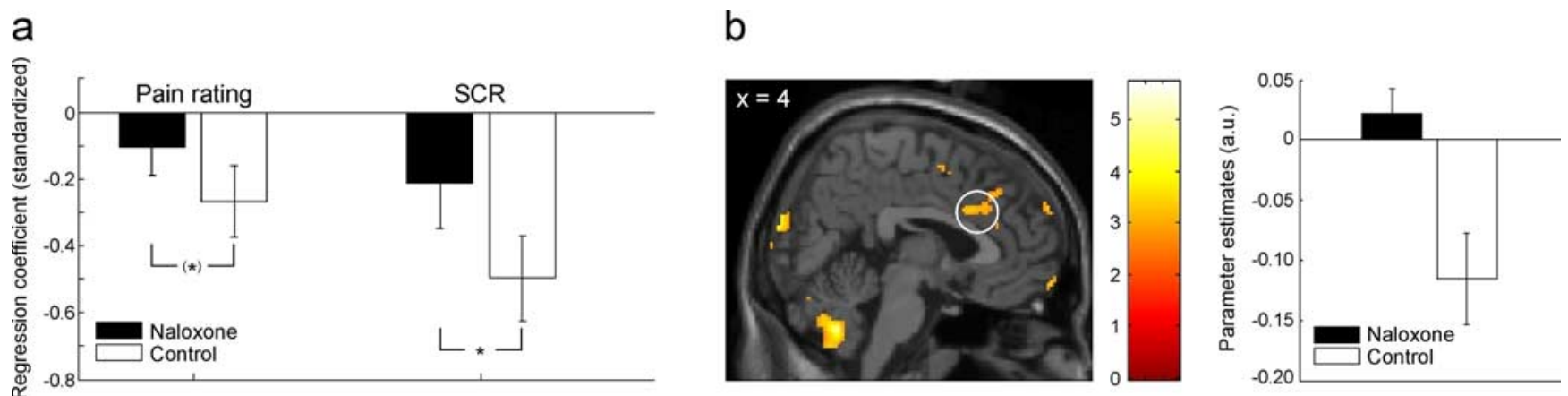

Figure 2. Habituation of responses to the US. $\boldsymbol{a}$, Habituating responses to the US on the behavioral level (pain ratings and SCR). For each subject, we calculated a regression with time as predictor and then compared the ensuing average regression coefficients of both groups. The bars in this graph thus represent the average regression coefficient of each group. The control group showed stronger habituation effects in the pain ratings (as reflected by a more negative average regression coefficient) than the naloxone group. In addition to this trend-level significant effect, the control group showed significantly stronger habituation effects in SCR to the US. $\boldsymbol{b}$, Habituating BOLD responses to the US. The dorsal anterior cingulate cortex showed significantly stronger habituating responses to the US in the control group than in the naloxone group. The bar graph on the right shows peak voxel parameter estimates that reflect the strength of habituation (i.e., the more negative, the stronger the habituation). The parameter estimates show that the BOLD responses in the control group habituate strongly, whereas the naloxone group shows sustained BOLD responses in dorsal anterior cingulate cortex. The image is thresholded at $p<0.005$ (uncorrected) for visualization purposes. ${ }^{*} p<0.05$. Error bars indicate SEM.

brain regions such as primary and secondary somatosensory cortex, insula, and thalamus (supplemental Table 1, available at www.jneurosci.org as supplemental material). Although there were no significant group differences in the pain ratings [naloxone, $67.12 \pm 4.25$, control, $63.88 \pm 5.54 ; t_{(28)}=0.46$; not significant (n.s.)], the naloxone group showed significantly stronger responses to the US in brain regions implicated in pain processing, including the insula [coordinates $x, y, z$ (in mm): 36, 20, 14; $\left.t_{(28)}=3.83 ; p=0.04\right]$, dorsolateral prefrontal cortex $(52,6,28$; $\left.t_{(28)}=3.76 ; p=0.04\right)$, and basal ganglia $\left(-16,10,0 ; t_{(28)}=3.37\right.$; $\left.p=0.04 ; 18,10,-2 ; t_{(28)}=3.20 ; p=0.05\right)$. Thus, our data provide only limited support for the suggestion that, with brief pain stimuli as used here, naloxone has a general hyperalgesic effect.

However, a learning-dependent effect of naloxone on US processing might be obscured by the simple categorical comparison performed above. More specifically, if a fearful state triggers the release of endogenous opioids and thereby leads to hypoalgesia, one might expect that such effects evolve over time as the subjects learn that the CS+ predicts the US [a notion that is also supported by the fact that in rats repeated trials are necessary for naloxone effects to emerge (Fanselow and Bolles, 1979)]. We therefore investigated whether the control group would show habituation in US responses over the course of the experiment that would be weaker or absent in the naloxone group. We observed that the control group showed stronger habituation in pain ratings $\left(t_{(28)}=1.24 ; p=0.11\right.$; trend level) and SCR to the US $\left(t_{(28)}=1.77 ; p=0.04\right)$ than the naloxone group (Fig. 2a). Mirroring these behavioral effects, we observed significantly stronger habituation effects in the control group in pain responsive brain regions, namely the dorsal anterior cingulate cortex [coordinates $x, y, z$ (in mm): $4,18,30 ; t_{(28)}=3.30 ; p=0.05$ ] (Fig. $\left.2 b\right)$ and insula $\left(36,0,16 ; t_{(28)}=4.16 ; p=0.02\right)$. Unexpectedly, we did not observe differential habituation effects in the amygdala. However, in a post hoc analysis using an exploratory threshold of $p<$ 0.005 (uncorrected), we observed strong habituation effects to the US in the amygdala across all subjects (main peak at 32, 4, $-18 ; t_{(28)}=5.51$ ), the parameter estimates of which showed a trend for greater habituation in the control group (supplemental Fig. 1 $a$, available at www.jneurosci.org as supplemental material). We then investigated the differential contrast with the same threshold and observed that the control group showed stronger habituation to the US in bilateral sublenticular extended amyg- dala $\left(-14,-2,-12 ; t_{(28)}=3.42 ; 20,-2,-6 ; t_{(28)}=2.80\right)($ supplemental Fig. 1b, available at www.jneurosci.org as supplemental material). Overall, the naloxone group thus showed more sustained responses to the US, which might be caused by a blockade of processing in a conditioning-dependent opioid-mediated antinociceptive circuit.

\section{CS-US association formation}

We therefore investigated whether the control group would show enhanced processing in a network implicated in descending pain control (Harris, 1996; Bingel et al., 2006) comprising the rACC, the amygdala, the PAG, and the RVM. Critically, if this network is indeed opioidergic and engaged by conditioning, then (1) processing in this network should be blocked in the naloxone group, (2) it should be engaged only on trials in which a US is expected (i.e., CS + trials), and (3) the response should be initiated before the US is administered. We thus investigated responses late in the CS phase ( $5 \mathrm{~s}$ after CS onset) and were interested in differential responses to CS+ vs CS - that differed between the two groups (i.e., a CS type by group interaction). We observed that in the $\operatorname{rACC}\left(10,32,8 ; t_{(28)}=4.24, p=0.01 ; 6,34,10 ; t_{(28)}=3.86, p=\right.$ $\left.0.02 ; 14,42,2 ; t_{(28)}=3.45, p=0.04\right)$, the amygdala $(-14,4,-22$; $\left.t_{(28)}=3.24 ; p=0.03\right)$, and the PAG $\left(0,-20,-6 ; t_{(28)}=2.84 ; p=\right.$ 0.06 ; trend level) there was a deactivation on CS + trials in the control group that was not evident on CS - trials and that was almost completely blocked in the naloxone group (Fig. 3). This response was initiated before the US was administered, as the time course shows (Fig. 3). Although the RVM did not show differential activity in the above analysis, there was significantly stronger coupling between the PAG and the RVM $\left(2,-32,-36 ; t_{(28)}=2.89 ; p=0.05\right)$ in the control group than in the naloxone group, as identified by a coupling by group analysis (Heinz et al., 2005) in which we used the PAG peak voxel as seed region.

Finally, we investigated whether the naloxone group would show signs of enhanced conditioning compared with the control group. On the behavioral level, we looked for a CS type by group interaction in SCR and RT data. Although we did not observe a significant interaction in SCR data $\left(F_{(1,27)}=0.68\right.$, n.s.), we observed a significant interaction in $\mathrm{RT}$ data $\left(F_{(1,28}=3.28 ; p=\right.$ $0.04)$ in that the naloxone group showed stronger conditioning effects (i.e., faster responses to CS + than to CS-) than the control group. With regard to the fMRI data, we were specifically 

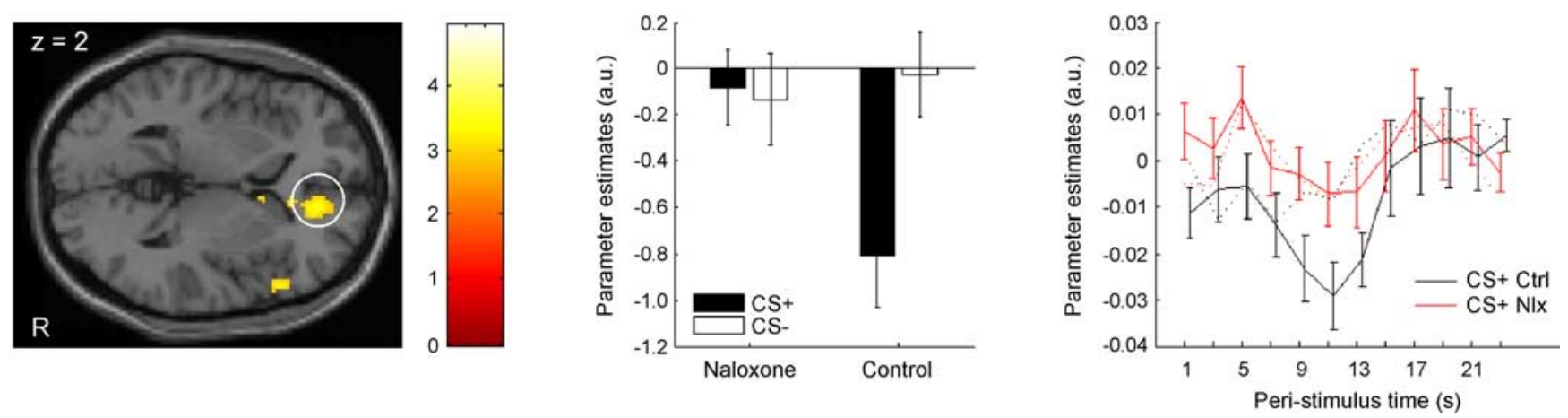

Figure 3. Conditioning induced responses in the antinociceptive system. The right ( $\mathrm{R}$ ) rostral anterior cingulate cortex shows a significant condition by group interaction late in the $C S$ interval (i.e., $5 \mathrm{~s}$ after (S onset; visualization threshold: $p<0.005$ uncorrected). As the parameter estimates in the bar graph show, this effect is mainly driven by a strong $C S+$ response in the control group that is almost completely blocked in the naloxone group; both groups show almost no response to the CS-. The parameter estimates indicate that the response to the CS+ in the control group is a deactivation, which we aimed to characterize further by examining the time course of this response. The rightmost panel thus shows the time course of the response on $C S+$ trials in the two groups (Ctrl, control group; NIx, naloxone group). The time course of the control group was shifted slightly to the right to avoid overlapping of error bars; time courses were obtained from an analysis that used FIRs as basis functions and does not make any assumptions about the shape of the hemodynamic response. Dotted lines show the time courses on CS - trials. The CS + time course of the control group shows a distinct deactivation, which starts at $\sim 5 \mathrm{~s}$ after $C S$ onset and ceases at $\sim 15 \mathrm{~s}$. Importantly, this deactivation is almost completely absent in the naloxone group. Error bars indicate SEM.
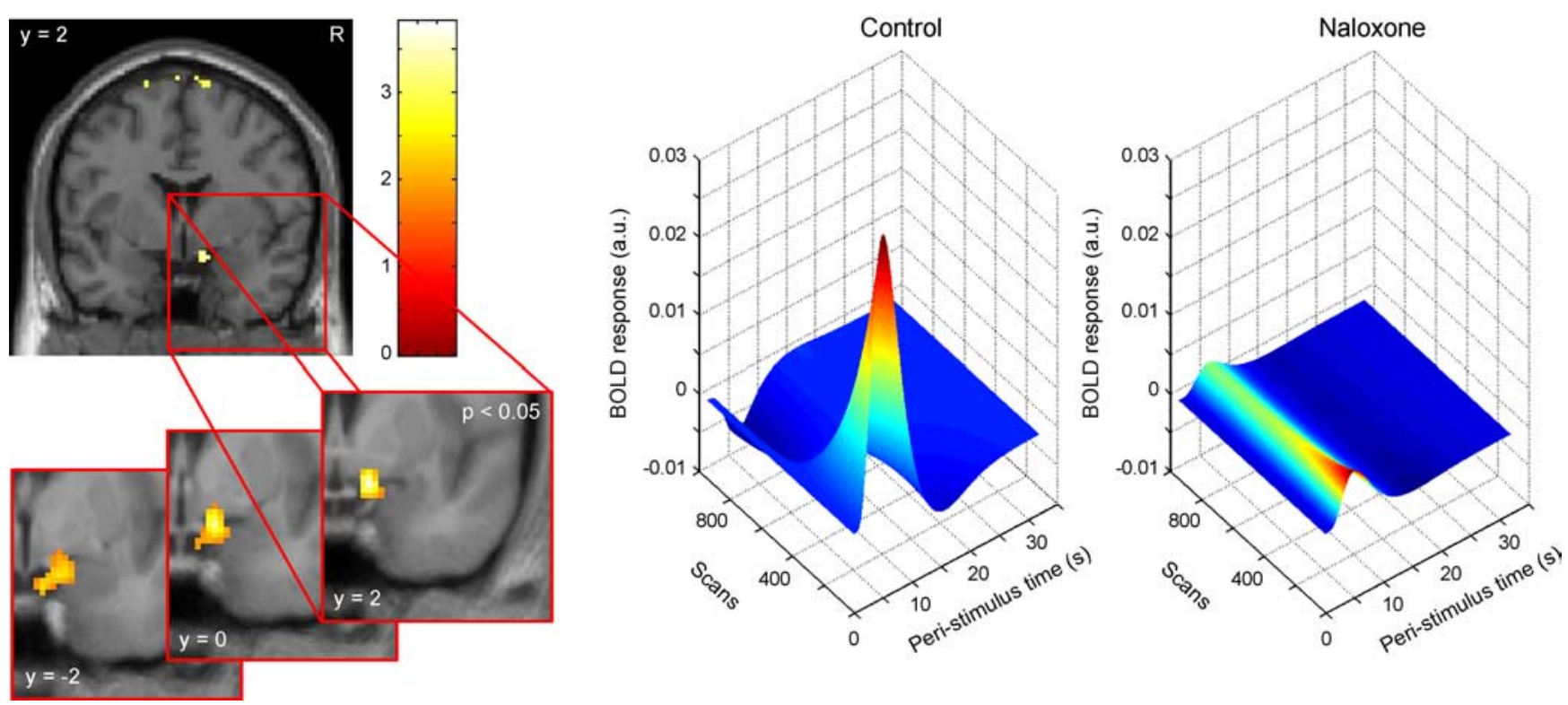

Figure 4. Conditioned amygdala responses at $\mathrm{CS}$ onset. The right (R) dorsomedial amygdala shows a significant time by condition by group interaction (visualization threshold: $p<0.005$ uncorrected). The insert uses a more liberal threshold and three slices of the averaged anatomical image of all subjects for additional characterization of the spatial extent and localization of this response. The three-dimensional plots on the right show the group-averaged fitted response in the amygdala to the CS + over the course of the experiment. The control group shows strongly decaying responses to the $C S+$ over time (i.e., decreasing responses along the axis labeled $S$ cans). In contrast, the naloxone group shows sustained responses over time.

interested in whether naloxone would block the often observed decay of conditioned amygdala responses over time (Quirk et al., 1997; Büchel et al., 1998; LaBar et al., 1998) and lead to sustained responses, as has been speculated previously (Büchel et al., 1998). We therefore tested whether in the control group the amygdala exhibits decreasing responses to the CS+ over time, which are significantly different from responses to the CS- (i.e., a time by condition interaction), and whether this pattern would be significantly different in the naloxone group. This analysis revealed significant activation in the right dorsomedial amygdala $(12,2$, $-18 ; t_{(28)}=3.13 ; p=0.04$ ) (Fig. 4). Most importantly, the control group showed decaying responses to the $\mathrm{CS}+$, whereas the naloxone group did not exhibit such decay and showed sustained responses to the CS+ (Fig. 4).

\section{Discussion}

In this study, we investigated the role of endogenous opioid neurotransmission in human fear conditioning. We observed that blocking opioidergic neurotransmission via naloxone inhibited the development of conditioned hypoalgesia, leading to more sustained responses to the US. Naloxone also blocked processing in a conditioning-dependent anticipatory antinociceptive network that includes areas with a high density of opioid receptors including rACC, amygdala and PAG. Finally, naloxone led to stronger conditioned responses and more sustained conditioning-dependent activity in the amygdala. Together, these results suggest that endogenous opioids have an inhibitory role in the acquisition of conditioned fear in humans.

Fanselow (1998) proposed a model of opioid function in fear 
conditioning, according to which convergence of CS and US information in the amygdala leads not only to the production of conditioned responses such as freezing, but also to a conditioned inhibitory signal mediated by opioids. This signal acts via brainstem structures such as PAG and RVM in an inhibitory way on ascending US input (the input that drives conditioning) thereby regulating fear conditioning in a negative-feedback manner. From this model, we derived three questions that we tested in human subjects. First, does naloxone inhibit the development of conditioned hypoalgesia (i.e., lead to more sustained US responses)? Second, does naloxone block the inhibitory signal that weakens US impact and thus causes conditioned hypoalgesia? Third, does naloxone lead to enhanced conditioning effects?

Previous research in rodents has shown that administration of opioid antagonists can block the development of conditioned hypoalgesia (Fanselow and Baackes, 1982; Helmstetter and Fanselow, 1987). Complementing these findings, we show that the control group showed stronger conditioned hypoalgesia, as indexed by nonsignificantly stronger habituation of pain ratings and significantly stronger habituation of SCR to the US. Mirroring these behavioral effects, the control group exhibited stronger habituation in BOLD responses in the $\mathrm{AACC}$ and the insula, the two regions most strongly implicated in pain processing (Apkarian et al., 2005). The activation in dACC corresponds very closely to a region specifically involved in coding perceived pain intensity (Büchel et al., 2002), providing additional evidence that perceived pain intensity decreased over time. Although this is in accord with the negative-feedback model, this model also requires that US input to the amygdala is diminished over time. Our data showed only a trend for stronger habituation in the amygdala in the control group, but in an extension of the amygdala proper, namely the sublenticular extended amygdala, we observed strongly habituating responses to the US in the control group but clearly sustained responses in the naloxone group.

The aforementioned model suggests that an amygdala/ brainstem-dependent circuit, which critically depends on opioidergic neurotransmission, implements conditioned hypoalgesia. Rodent studies have shown that injection of $\mu$-opioid, but neither $\delta$ - nor $\kappa$-opioid, antagonists into the amygdala, the PAG, and the RVM can inhibit the production of conditioned hypoalgesia (Bellgowan and Helmstetter, 1998; Foo and Helmstetter, 1999). In addition to these regions, we also focused on the rACC, which is involved in placebo analgesia, a form of endogenous pain control that strongly involves the opioidergic system (Petrovic et al., 2002; Zubieta et al., 2005; Wager et al., 2007). We observed that the control group showed responses in this antinociceptive network, namely in the rACC, amygdala, and PAG, that were almost completely blocked in the naloxone group. These responses were specifically related to conditioning, as they only occurred on CS + , but not on CS - , trials and started before the US was administered. The presence of the CS + is thus sufficient to engage an opioidergic antinociceptive network in preparation for the arrival of the US. The responses in the control group were deactivations, which is particularly interesting, because one way of opioidergic neurotransmission is postsynaptic inhibition (Faber and Sah, 2004) and there is evidence that negative BOLD responses reflect decreased neuronal activity (Shmuel et al., 2006), at least in cortical structures. The deactivation to the CS+ in the rACC is also consistent with results from both fMRI studies (Phelps et al., 2004; Milad et al., 2007) and recordings from rodent infralimbic cortex which is an apparent homolog of human rACC (Santini et al., 2008). Interestingly, the RVM did not show differential responses, but showed stronger coupling with the
PAG in the control group, consistent with an opioidergic innervation of the RVM via the PAG (Fields, 2004). Thus, blockade of opioid neurotransmission also seems to block the connectivity of regions implicated in descending pain control.

Finally, Fanselow's (1998) model suggests that blocking opioidergic neurotransmission leads to stronger conditioning effects. Numerous studies have shown that opioid agonists attenuate (Davis, 1979; Westbrook et al., 1991) and opioid antagonists facilitate (Westbrook et al., 1991; Young and Fanselow, 1992; McNally et al., 2004a) the acquisition of conditioned fear. In line with this, we observed stronger conditioned responses in the naloxone group as evidenced by differential reaction times [which have been used previously as an index of conditioning (Seymour et al., 2005)]: subjects reacted faster to the CS+ than to the CS-, possibly because of stronger attentional engagement to a stimulus that predicts pain (Damme et al., 2004). Because the US has a stronger impact in the naloxone group, the CS signaling the US might consequently also engage more attention. We observed no enhancement of conditioning in SCR, although there was a main effect of conditioning across all subjects, showing that SCR were sensitive to conditioning. This null finding cannot be the result of SCR being insensitive to naloxone effects because differential habituation to the US was clearly evident in SCR across the two groups, but might instead be attributable to low statistical power, because only half of all CS + trials could be included in the analysis.

With regard to a neural locus of enhanced conditioning effects, we investigated responses in the amygdala. Previous studies demonstrated conditioning-induced amygdala activation that decays rapidly across conditioning trials (Quirk et al., 1997; Büchel et al., 1998; LaBar et al., 1998). It has been speculated that this decay might be caused by an opioidergic negative feedback signal that over time reduces the ability of the US to support conditioning (Büchel et al., 1998). Blocking the negative feedback signal via naloxone should thus lead to sustained conditioned amygdala activation. In line with these suggestions, we observed that the control group showed a decaying response profile in the amygdala that was specific to the CS+. More importantly, the naloxone group did not show such decay, but exhibited sustained responses to the $\mathrm{CS}+$. We suggest that this decay relates to a prediction error-like signal that is re-established at trial onset to compute the current prediction. In line with this, it has been shown that the amygdala encodes predictions in aversive learning (Gläscher and Büchel, 2005) and coexpression of predictions and prediction errors in the same brain structure has been observed previously (Yacubian et al., 2006). In the control group, the prediction error decreases rapidly because of the inhibitory opioidergic signal causing conditioned hypoalgesia, whereas in the naloxone group where the inhibitory opioidergic signal is blocked, its decrease is much less pronounced. Unexpectedly, the fitted responses to the $\mathrm{CS}+$ in the amygdala differed at the beginning of conditioning. We refrain from interpreting this shift in absolute value because this would necessitate single trial and trial history modeling, but speculate that it might possibly be attributable to unspecific drug effects or other gain-related processes in the amygdala.

Although our results support many predictions derived from Fanselow's (1998) model, some limitations of the present study are worth mentioning. First, we cannot rule out that naloxone also influenced attentional (Arnsten et al., 1984; Iordanova et al., 2006) or memory processes (McGaugh et al., 1988). Second, we only studied male subjects and, thus, cannot ascertain whether the observed effects can be generalized to females, as there are 
gender differences in both fear conditioning and opioid function (Zubieta et al., 2002; Milad et al., 2006). Third, we cannot distinguish between naloxone influences on the acquisition versus the expression of conditioned fear. Finally, based on neurophysiological simulations it was suggested previously that opioids cannot exert their negative feedback function alone, but need to work in concert with a faster-acting inhibitory neurotransmitter such as GABA (Padlubnaya et al., 2006).

One question that remains is why endogenous opioids are engaged in conditioned fear. It was suggested that release of endogenous opioids during fear serves to make an organism less sensitive to pain and therefore allows more resources to be devoted to coping with the immediate threat (Bolles and Fanselow, 1980). Intriguingly, in conditioned fear, an initially neutral stimulus is able to engage this antinociceptive opioid response. In contrast to this explanation, McNally and colleagues (McNally et al., 2004b; McNally and Cole, 2006) suggested that endogenous opioids are not exclusively involved in procedures that involve noxious stimulation, but that they have a more general role in regulating prediction errors during aversive learning. This could explain facilitation of conditioned fear responses by naloxone (Young and Fanselow, 1992; McNally et al., 2004a), impairment of fear extinction by naloxone (McNally and Westbrook, 2003; McNally et al., 2004b) and naloxone facilitation/morphine depression of conditioned responses in paradigms in which no noxious US is administered (Mauk et al., 1982a,b; Cicala et al., 1990). Our results do not allow dissociating between the two models, for which it would be necessary to investigate other forms of learning such as second order fear conditioning or extinction of conditioned fear. Note however, that the model of McNally and colleagues (McNally et al., 2004b; McNally and Cole, 2006) also predicts the naloxone-dependent enhancement of conditioned fear observed in this study. Thus, there might be dual routes via which opioids act in aversive learning: one attenuating US impact via conditioned hypoalgesia and one directly regulating prediction errors.

\section{References}

Amanzio M, Benedetti F (1999) Neuropharmacological dissection of placebo analgesia: expectation-activated opioid systems versus conditioning-activated specific subsystems. J Neurosci 19:484-494.

Apkarian AV, Bushnell MC, Treede RD, Zubieta JK (2005) Human brain mechanisms of pain perception and regulation in health and disease. Eur J Pain 9:463-484.

Arnsten AF, Neville HJ, Hillyard SA, Janowsky DS, Segal DS (1984) Naloxone increases electrophysiological measures of selective information processing in humans. J Neurosci 4:2912-2919.

Bellgowan PS, Helmstetter FJ (1998) The role of mu and kappa opioid receptors within the periaqueductal gray in the expression of conditional hypoalgesia. Brain Res 791:83-89.

Bingel U, Lorenz J, Schoell E, Weiller C, Büchel C (2006) Mechanisms of placebo analgesia: rACC recruitment of a subcortical antinociceptive network. Pain 120:8-15.

Bingel U, Schoell E, Herken W, Büchel C, May A (2007) Habituation to painful stimulation involves the antinociceptive system. Pain 131:21-30.

Bolles RC, Fanselow MS (1980) A perceptual-defensive-recuperative model of fear and pain. Behav Brain Sci 3:291-323.

Büchel C, Morris J, Dolan RJ, Friston KJ (1998) Brain systems mediating aversive conditioning: an event-related fMRI study. Neuron 20:947-957.

Büchel C, Bornhovd K, Quante M, Glauche V, Bromm B, Weiller C (2002) Dissociable neural responses related to pain intensity, stimulus intensity, and stimulus awareness within the anterior cingulate cortex: a parametric single-trial laser functional magnetic resonance imaging study. J Neurosci 22:970-976.

Calcagnetti DJ, Helmstetter FJ, Fanselow MS (1987) Quaternary naltrexone reveals the central mediation of conditional opioid analgesia. Pharmacol Biochem Behav 27:529-531.
Cicala GA, Azorlosa JL, Estall LB, Grant JS (1990) Endogenous opioids interfere with pavlovian second-order fear conditioning. Psychol Sci $1: 312-315$

Damme SV, Lorenz J, Eccleston C, Koster EH, Clercq AD, Crombez G (2004) Fear-conditioned cues of impending pain facilitate attentional engagement. Neurophysiol Clin 34:33-39.

Davis M (1979) Morphine and naloxone: effects on conditioned fear as measured with the potentiated startle paradigm. Eur J Pharmacol 54:341-347.

Faber ES, Sah P (2004) Opioids inhibit lateral amygdala pyramidal neurons by enhancing a dendritic potassium current. J Neurosci 24:3031-3039.

Fairhurst M, Wiech K, Dunckley P, Tracey I (2007) Anticipatory brainstem activity predicts neural processing of pain in humans. Pain 128:101-110.

Fanselow MS (1998) Pavlovian conditioning, negative feedback, and blocking: mechanisms that regulate association formation. Neuron 20:625-627.

Fanselow MS, Baackes MP (1982) Conditioned fear-induced opiate analgesia on the Formalin test: Evidence for two aversive motivational systems. Learn Motiv 13:200-221.

Fanselow MS, Bolles RC (1979) Naloxone and shock-elicited freezing in the rat. J Comp Physiol Psychol 93:736-744.

Fanselow MS, Calcagnetti DJ, Helmstetter FJ (1988) Peripheral versus intracerebroventricular administration of quaternary naltrexone and the enhancement of pavlovian conditioning. Brain Res 444:147-152.

Fields H (2004) State-dependent opioid control of pain. Nat Rev Neurosci 5:565-575.

Foo H, Helmstetter FJ (1999) Hypoalgesia elicited by a conditioned stimulus is blocked by a mu, but not a delta or a kappa, opioid antagonist injected into the rostral ventromedial medulla. Pain 83:427-431.

Foo H, Westbrook RF (1994) The form of the conditioned hypoalgesic response resulting from preexposure to a heat stressor depends on the pain test used. Psychobiology 22:173-179.

Friston KJ, Buechel C, Fink GR, Morris J, Rolls E, Dolan RJ (1997) Psychophysiological and modulatory interactions in neuroimaging. NeuroImage 6:218-229.

Gläscher J, Büchel C (2005) Formal learning theory dissociates brain regions with different temporal integration. Neuron 47:295-306.

Good AJ, Westbrook RF (1995) Effects of a microinjection of morphine into the amygdala on the acquisition and expression of conditioned fear and hypoalgesia in rats. Behav Neurosci 109:631-641.

Gottfried JA, Dolan RJ (2004) Human orbitofrontal cortex mediates extinction learning while accessing conditioned representations of value. Nat Neurosci 7:1144-1152.

Gutstein HB, Akil H (2006) Goodman and Gilman's the pharmacological basis of therapeutics (Brunton LL, Lazo JS, Parker KL, eds), pp 547-590. New York: McGraw-Hill.

Harris JA (1996) Descending antinociceptive mechanisms in the brainstem: their role in the animal's defensive system. J Physiol (Paris) 90:15-25.

Heinz A, Braus DF, Smolka MN, Wrase J, Puls I, Hermann D, Klein S, Grüsser SM, Flor H, Schumann G, Mann K, Büchel C (2005) Amygdalaprefrontal coupling depends on a genetic variation of the serotonin transporter. Nat Neurosci 8:20-21.

Helmstetter FJ, Fanselow MS (1987) Effects of naltrexone on learning and performance of conditional fear-induced freezing and opioid analgesia. Physiol Behav 39:501-505.

Iordanova MD, McNally GP, Westbrook RF (2006) Opioid receptors in the nucleus accumbens regulate attentional learning in the blocking paradigm. J Neurosci 26:4036-4045.

Knight DC, Nguyen HT, Bandettini PA (2003) Expression of conditional fear with and without awareness. Proc Natl Acad Sci USA 100:15280-15283.

LaBar KS, Gatenby JC, Gore JC, LeDoux JE, Phelps EA (1998) Human amygdala activation during conditioned fear acquisition and extinction: a mixed-trial fMRI study. Neuron 20:937-945.

LeDoux JE (2000) Emotion circuits in the brain. Annu Rev Neurosci 23:155-184.

Maren S, Quirk GJ (2004) Neuronal signalling of fear memory. Nat Rev Neurosci 5:844-852.

Mauk MD, Warren JT, Thompson RF (1982a) Selective, naloxonereversible morphine depression of learned behavioral and hippocampal responses. Science 216:434-436.

Mauk MD, Madden J, Barchas JD, Thompson RF (1982b) Opiates and clas- 
sical conditioning: selective abolition of conditioned responses by activation of opiate receptors within the central nervous system. Proc Natl Acad Sci USA 79:7598-7602.

McGaugh JL, Introini-Collison IB, Nagahara AH (1988) Memoryenhancing effects of posttraining naloxone: involvement of betanoradrenergic influences in the amygdaloid complex. Brain Res 446:37-49.

McNally GP, Cole S (2006) Opioid receptors in the midbrain periaqueductal gray regulate prediction errors during pavlovian fear conditioning. Behav Neurosci 120:313-323.

McNally GP, Westbrook RF (2003) Opioid receptors regulate the extinction of pavlovian fear conditioning. Behav Neurosci 117:1292-1301.

McNally GP, Pigg M, Weidemann G (2004a) Blocking, unblocking, and overexpectation of fear: a role for opioid receptors in the regulation of pavlovian association formation. Behav Neurosci 118:111-120.

McNally GP, Pigg M, Weidemann G (2004b) Opioid receptors in the midbrain periaqueductal gray regulate extinction of pavlovian fear conditioning. J Neurosci 24:6912-6919.

Milad MR, Goldstein JM, Orr SP, Wedig MM, Klibanski A, Pitman RK, Rauch SL (2006) Fear conditioning and extinction: influence of sex and menstrual cycle in healthy humans. Behav Neurosci 120:1196-1203.

Milad MR, Wright CI, Orr SP, Pitman RK, Quirk GJ, Rauch SL (2007) Recall of fear extinction in humans activates the ventromedial prefrontal cortex and hippocampus in concert. Biol Psychiatry 62:446-454.

Padlubnaya DB, Parekh NH, Brown TH (2006) Neurophysiological theory of kamin blocking in fear conditioning. Behav Neurosci 120:337-352.

Petrovic P, Kalso E, Petersson KM, Ingvar M (2002) Placebo and opioid analgesia: imaging a shared neuronal network. Science 295:1737-1740.

Phelps EA, Delgado MR, Nearing KI, LeDoux JE (2004) Extinction learning in humans: role of the amygdala and vmPFC. Neuron 43:897-905.

Pitman RK, van der Kolk BA, Orr SP, Greenberg MS (1990) Naloxonereversible analgesic response to combat-related stimuli in posttraumatic stress disorder. A pilot study. Arch Gen Psychiatry 47:541-544.

Quirk GJ, Armony JL, LeDoux JE (1997) Fear conditioning enhances different temporal components of tone-evoked spike trains in auditory cortex and lateral amygdala. Neuron 19:613-624.

Santini E, Quirk GJ, Porter JT (2008) Fear conditioning and extinction dif- ferentially modify the intrinsic excitability of infralimbic neurons. J Neurosci 28:4028-4036.

Seymour B, O’Doherty JP, Koltzenburg M, Wiech K, Frackowiak R, Friston K, Dolan R (2005) Opponent appetitive-aversive neural processes underlie predictive learning of pain relief. Nat Neurosci 8:1234-1240.

Shmuel A, Augath M, Oeltermann A, Logothetis NK (2006) Negative functional MRI response correlates with decreases in neuronal activity in monkey visual area V1. Nat Neurosci 9:569-577.

Sprenger T, Valet M, Boecker H, Henriksen G, Spilker ME, Willoch F, Wagner KJ, Wester HJ, Tölle TR (2006) Opioidergic activation in the medial pain system after heat pain. Pain 122:63-67.

Tepperman FS, Hirst M, Smith P (1983) Brain and serum levels of naloxone following peripheral administration. Life Sci 33:1091-1096.

Wager TD, Scott DJ, Zubieta JK (2007) Placebo effects on human muopioid activity during pain. Proc Natl Acad Sci USA 104:11056-11061.

Westbrook RF, Greeley JD, Nabke CP, Swinbourne AL (1991) Aversive conditioning in the rat: effects of a benzodiazepine and of an opioid agonist and antagonist on conditioned hypoalgesia and fear. J Exp Psychol Anim Behav Process 17:219-230.

Willer JC, Dehen H, Cambier J (1981) Stress-induced analgesia in humans: endogenous opioids and naloxone-reversible depression of pain reflexes. Science 212:689-691.

Wolter J, Lachnit H (1993) Are anticipatory first and second interval skin conductance responses indicators of predicted aversiveness? Integr Physiol Behav Sci 28:163-166.

Yacubian J, Gläscher J, Schroeder K, Sommer T, Braus DF, Büchel C (2006) Dissociable systems for gain- and loss-related value predictions and errors of prediction in the human brain. J Neurosci 26:9530-9537.

Young SL, Fanselow MS (1992) Associative regulation of pavlovian fear conditioning: unconditional stimulus intensity, incentive shifts, and latent inhibition. J Exp Psychol Anim Behav Process 18:400-413.

Zubieta JK, Smith YR, Bueller JA, Xu Y, Kilbourn MR, Jewett DM, Meyer CR, Koeppe RA, Stohler CS (2002) $\mu$-opioid receptor-mediated antinociceptive responses differ in men and women. J Neurosci 22:5100-5107.

Zubieta JK, Bueller JA, Jackson LR, Scott DJ, Xu Y, Koeppe RA, Nichols TE Stohler CS (2005) Placebo effects mediated by endogenous opioid activity on $\mu$-opioid receptors. J Neurosci 25:7754-7762. 